\title{
Comparison of the effect of game based on educational method and traditional approach on the performance of selected basketball skills
}

Ghanati P. ${ }^{1 \mathrm{ABCDE}}$, MohammadZadeh H. ${ }^{2 \mathrm{ABCDE}}$

${ }^{1}$ Department of Physical Education and Sport Sciences, Urmia University, Iran

${ }^{2}$ Department of Physical Education and Motor Behavior, Urmia University, Iran

Authors' Contribution: A - Study design; B - Data collection; C - Statistical analysis; D - Manuscript Preparation; E - Funds Collection.

\begin{abstract}
Purpose: $\quad$ The purpose of this study was to investigate the effect of the game based on educational method and traditional approach on the performance of selected basketball skills.

Material: $\quad$ The type of research was semi-experimental one. Participants included 30 adolescent girls who were divided into two groups based on the game-based practice (15) and traditional training (15) build on the pre-test scores. Both groups performed the intervention program for 8 weeks and each week for three 60 -minute sessions. Then, a posttest was performed and the data were analyzed using SPSS 21 software at a significance level of 0.05 .

Results: $\quad$ The results showed that both educational method and traditional approach groups had a significant improvement in basketball performance; there was no improvement in basketball dribbling performance in both groups. However, in the performance test, game based on educational method group had a significant improvement compared to the traditional practice one.

Conclusions: The results suggest that using a game-based educational method can significantly increase the important factors of basketball performance in youth, which can move into more complex situations.

Keywords: game, educational method, traditional practice, basketball, performance.
\end{abstract}

\section{Introduction}

Team sports can play an important role in attracting youth to sports and continuing their activities. But teens do not enjoy exercises and have little physical mobility when participating in team sports. Many of them do not know how and to which area of the field move, the traditional educational models in the physical education classes of the universities may be an effective factor in reducing the quality of play in team sports. As Butler \& Griffin argued, as a result of the use of traditional models, in addition to children, adults also find little understanding of the game [1]. The traditional method of training and upgrading performance emphasizes the use of a teachercentered technique and approach, the coach is the main controller of the learning environment and responsible for the content planning of the course [2, 3], the skills are practiced in a drill and distinct form from the real context. They are practiced in such a way that the learner focuses on the repetition of skill execution shown to him by the pattern [2]. Pritchard et. al. stated that one of the most important limitations of traditional models is the disability of transferring the practicing skills to the real field of play, resulting in a significant lack of performance in the game performance. Ultimately, using this approach leads to boredom and fatigue [4]. As a result of these observations, different educational approach, called PPbased learning, was developed by Bunker and Thorpe [5]. The goal of the game's learning approach is to develop an understanding of the game insight, which makes the performer more confident.

Therefore, the main purpose of PP is understanding and appreciation of the game [6]. Advocates of the PP approach propose that action or decision-making choices should be taught before the practice or execution of the (c) Ghanati P., MohammadZadeh H., 2018

doi:10.15561/20755279.2018.0402 technique [7]. In fact, achieving the objectives of the PP approach is based on modified games and questioning [8]. Some researchers emphasize the valuable role played by sport education and order physical education instructors to provide educational issues in game tutorials. He also insists that coaches "Turn Practice into Play". The change in practice to play has two kinds of beneficial effects: motivation and transfer $[9,10]$. The motivational aspect of the game based educational model comes from the enjoyment of the game and the perceived progress of learning. The PP model replaces the training's tedious condition with the challenging and enjoyable playing situations. In addition, if the learner overcomes the problems and challenges, he will feel progress and enjoyment. In general, enjoyment is the most important factor in the participation of children and youth in sport. Enjoyment is the main reason for the beginning and participation in the sport of youth, the researchers concluded that enjoyment of sport activities would increase the commitment of youth to exercise and physical activity and reduce interest in alternative activities, such as watching TV subsidies.

After the introduction of the PP method and other tactic-based models, several studies have been done on their impact on the growth of technique and performance of the game for children and youth. In some studies, gamebased or tactical groups based on post-training techniques did not show significant differences in skill performance $[8,11]$ and some reported superiority of the game-based group [4, 12, 13]. In the area of decision making, the result of the research was contradictory. Research in some respects supported the relative superiority of the tactic group $[8,10,13]$ and in some other aspects did not show such superiority $[14,15]$. Further research supports the superiority of tactic training $[4,8,16]$. Miller in a review 
of research on tactical models reported that $56 \%$ of the development studies, $58 \%$ of decision-making studies, $93 \%$ of support development studies and $59 \%$ of the studies supported of skill development [15]. Regardless of the support factor, these findings are consistent with what Lee and Ward stated [17]. According to them, research on tactical models has provided little support for cognitive outcomes and game performance, and there is still little empirical evidence that supports tacticbased approaches [17]. But according to McMorris, the learner learns what they are taught [18]. Game-based learning leads to the learning and improvement of tactical performance, and the teaching of techniques leads to learning and technical progress. McMorris also concludes that evidence suggests that technology-based learning leads to decision-making, and game-based learning may develop technical performance [18-20]. Miller suggests that long training courses (more than 8 hours) should be used in research [15]. Miller concluded that a longer-term training course had a great influence on progress and promotion of decision-making skills [15].

Also, Harvey and Jarrett, after reviewing the studies conducted since 2006, concluded that the length of the training period for research on game-based approaches is still low. They stated that the length of the intervention period of the research was usually seven to twelve sessions [21]. However, the length of the intervention period was appropriate in the research of Lee and Enter [17], Mesquita et. al. [13], and Pritchard et. al. [4]. But it seems that the only limited number of training sessions in most of the comparative studies is not important. McMurray [22] further criticized the comparative studies of the game-based approach with the traditional approach. McMorris states on the basis of the motor behavior approach that proposes a motor behavior for a techniqueto-cognitive approach [18]. He claims that what they criticize of the game-based approach call it the "traditional approach" are poorly-documented training protocols that are different from the technique-to-cognitive approach [18]. Similarly, Chatzopoulos et al. put forward a similar critique, according to them, what has not been considered is the continuation of a technique based approach using tactical strategies [11]. Given what has been said, the issues that have been neglected in previous research have been to prolong the course and, consequently, change the traditional approach of the classroom (ie, technique to tactics), as well as the ability to transfer learning to the actual field of play. Investigations have shown that with the increase in the training period, the results also change [15]. By increasing the number of sessions, the type of traditional group training will also change, and their training will change from the state modified technique to more complex situations, such as moderated games. But other features of the traditional approaches, namely, coach-centered, providing direct instructions and coaching decisions are still there.

The purpose of this study is to compare the effect of two educational methods, namely game based (PP) and traditional, on the performance of basketball.

\section{Materials and Methods}

Participants.

Participants included 30 teenage girls from Urmia who volunteered to participate in this research. None of the participants had a background of basketball practice. Participants were randomly divided into two equal groups (15 subjects). A group based on the practice-based training, the other group trained in the traditional way.

\section{Research Design}

The research method was semi-experimental, in which two groups participated. Participants were evaluated in two stages of pre-test and post-test. The participants requested to participate in the research after completing the consent, of which 45 were participants in the pretest of basketball performance. The evaluations were carried out using the game performance assessment instrument measurement tool, based on three scales of shot, dribbling and pass. Participants' scores were used for homogenization. 8 skilled participants, as well as 7 participants who appeared to have motion problems, were removed at this stage. Finally, 30 participants with a skill level of approximately equal were selected. For the PP group, a trainer with a 10-year history of basketball training and a trainer with eleven years of basketball training experience were used for the traditional group.

In addition, three first-rate basketball coaches with a history of training and coaching participated as research consultants. The practice protocol of each group was designed according to the general pattern of the model, using similar research and the opinion of the counselor's mentors. Before each training session, information about the content of the lesson was given to the training coach. The groups trained for 60 minutes each week for six weeks and each session for 60 minutes each day after the last training session, a post-test (similar to the pre-test). Assessments of decision making, skill play and game support simultaneously were conducted by two coaches who were familiar with the performance test.

The percentage of agreement between the two evaluators was $82 \%$ for decision making, $87 \%$ for skill and $84 \%$ for support. The design of the PP group exercise program was done using the extended PP model [21, 22]. To design a traditional group training program, two distinctive features of the traditional approach, namely, teacher-centered and technically based, were considered. The group's training program at initial meetings included drilling of techniques such as dribbling, pass and a variety of shots. Drilling the techniques was done individually and by team. The instructor used direct instructions, modeling and feedback for training. At the end of each training session, the participant played basketball for 1015 minutes freely.

After the ninth session, after moderating progress in the execution of the techniques, moderated games were used to teach skill-utilization in challenging situations. Moderated games were also used to train attack and defense tactics such as outlet, counter-strike, regional defense. The complexity of the exercises increased gradually throughout the sessions. The main difference 
between the use of moderated games in the traditional group and PP focused on the characteristics of the two approaches. For the traditional team, even after the ninth session and starting to practice moderated games, the coach played a pivotal role in all decisions. The coach guided the game with instructions, guides and concurrent feedback. The question was not used about how to freeze the game and what is better for a player to do.

The participants tried to execute the coach's instructions in the form of the game. Game performance assessment instrument was designed by Oslin et al. to measure game performance $[25,26]$. The mean of play performance is behaviors that represent the understanding of the game as well as the player's ability to solve tactical problems through the selection and application of appropriate skills. The instrument for measuring the performance of the game has been examined by three groups of games: attack (football and basketball), net/ wall (volleyball) and field/track/points (softball). The validity and reliability of the test were measured through three separate studies on guidance students, and the findings have shown that this tool is a valid and reliable test for measuring the game performance [26]. This tool was used to measure three indicators of decision making, skill execution and support. According to Oslin et al., these three indicators are important for success in assault games such as basketball [26].

Statistical Analysis

To investigate the present research data and compare the effect of two methods of training with traditional approach and game-based learning on the performance of selected basketball skills, first, the effect of each two methods in three skills was calculated by t-test and then for comparison of these methods effects in each three skills single-variable covariance analysis (ANCOVA) was used. Data were analyzed with confidence interval of $0.95(\mathrm{P}=0.05)$ and analyzed by SPSS software version 22.

\section{Results}

Shapiro-Wilk test was used to examine the distribution of research data. The results showed that the data of all three skills in the two stages of the test were normal distribution $(\mathrm{P}>0.05)$, therefore, for statistical analysis, parametric statistical method was used. In order to investigate the effect of traditional and game-based training on basketball triple skills in the present study, the scores of each two groups were compared in the pre-test and performance test, the results of which are shown in the following table.

TAs the results of the test show (Table 1) in basketball skill scoring, both educational methods have made significant progress among the participants in the field, so that the scores from the pre-test to the performance test were significantly improved $(\mathrm{P}=0.001)$. In the case of dribbling skills, the difference between the two stages of the test (pre-test and performance test) was statistically significant $(\mathrm{P}<0.05)$. However, with regard to mean of two groups in two stages of test, it is observed that two groups influenced by independent variable (Educational methods) had worse performance at the test stage than the pre-test. But the results on the pass variable are different, so that there is no significant improvement in the traditional educational group among the subjects, and the traditional teaching method has no significant effect on improving of pass technique $(\mathrm{P}=0.054)$, but in the training group of the based game, the results indicate that the progress of the subjects from the pre-test stage to the performance test is statistically significant $(\mathrm{P}=0.001)$; In other words, game based education has a significant effect on the pass technique of the subjects in the present study.

In order to compare the effects of two educational methods on the basketball skills of this study, onevariable covariance analysis (ANCOVA) was used. The results are shown in the tables below. First, to compare the skill of the shot between the two groups (two educational methods), we examine the results of this test in Table 2. At first, the assumption of the equality of variances was confirmed by Levin's test for all three skills ( $\mathrm{P}>0.05)$.

The results of the test in the above table show that the $F$ value of the coincidence variable (pretest) is significant $(\mathrm{P}=0.001, \mathrm{P}=13.072)$, therefore, hypothesis correlation assumption is observed and choice of pre-test score as a perfect match. But value of independent variable F (group)

Table 1. The Effect of Two Methods of Teaching on Performance in Basketball Skills

\begin{tabular}{|c|c|c|c|c|c|}
\hline Group & Skills & Test & $M$ & $T$ & Sig. \\
\hline & Shot & pretest & 16.26 & -5.9 & 0.001 \\
\hline & & Performance & 20.87 & & \\
\hline Traditional & Dribbling & pretest & 12.16 & 3.5 & 0.004 \\
\hline \multirow[t]{4}{*}{ Approach } & & Performance & 11.14 & & \\
\hline & Pass & pretest & 26.93 & -2.1 & 0.054 \\
\hline & & Performance & 28.93 & & \\
\hline & Shot & pretest & 17.2 & -4.58 & 0.001 \\
\hline Game Based & & Performance & 20.47 & & \\
\hline Educational & Dribbling & pretest & 12.26 & 4.71 & 0.001 \\
\hline \multirow[t]{3}{*}{ Method } & & Performance & 11.06 & & \\
\hline & Pass & pretest & 24.2 & -5.31 & 0.001 \\
\hline & & Performance & 35.73 & & \\
\hline
\end{tabular}


(educational method) is not statistically significant ( $\mathrm{P}$ $=0.338, \mathrm{~F}=0.952$ ). After removing the impact of pretest, there is no significant difference between the mean changes of two groups in the post-test. Therefore, we conclude that there is no significant difference between the two methods of teaching (traditional and game based) on the performance of basketball shots.

The results of the ANCOVA test for dribbling skills are shown in Table 3.

The results of the test in the above table indicate that the $\mathrm{F}$ value of the co-variance (pre-test) is significant $(\mathrm{P}=0.001, \mathrm{~F}=106.329)$, therefore, correlation assumption of coincidence variable is observed and the choice of pretest score as a perfect match. But value of independent variable $\mathrm{F}$ (group) (educational method here) is not statistically significant $(\mathrm{P}=0.632, \mathrm{~F}=234)$. After removing the impact of pre-test, there is no significant difference between the mean changes of the two groups in the post-test. Therefore, we conclude that there is no significant difference between the two educational methods (traditional and game-based) on performance of basketball dribbling skill in the present study.

For the skill level, ANCOVA test results are shown in Table 4.

The results of the test in the above table show that the $\mathrm{F}$ value of the co-variance (pretest) is significant $(\mathrm{P}=0.001$, $\mathrm{F}=4.147$ ), therefore, correlation assumption of convergent variable is observed and the choice of the pre-test score as a perfect match. The value of independent variable
F (group) (educational method here) is statistically significant $(\mathrm{P}=0.001, \mathrm{~F}=143.14)$. After removing effect of pre-test, there is a significant difference between the mean variations of two groups in performance test. Therefore, we conclude that there is a significant difference between two methods of teaching (traditional and game based) on pass execution of subjects in the present study. According to the mean scores of the two groups, we conclude that the method of education based on the game had a more significant effect on pass performance of subjects than traditional teaching method. In general, the results of this study showed the game-based method of training compared to the traditional method significantly had a greater effect on pass performance of subjects, but there was no significant difference between the two methods of training on shots and dribbling skills.

\section{Discussion}

The purpose of this study was to investigate the effect of two approaches based on the game (PP) and traditional training on the performance of selected youth girls' basketball skills. Participants practiced basketball in two traditional and PP groups in 18 sessions. The results were evaluated in the form of pre-test and post-test.

Overall, the results show that subjects in both traditional training and game-based training played a significant role in basketball shots, but there was no significant improvement in dribbling, but in gamebased training test they had meaningful progress in pass

Table 2. Results of the covariance analysis test to examine the effect of two educational methods on the skill of basketball shots

\begin{tabular}{llllll}
\hline Source & SS & df & MS & F & Sig. \\
\hline Correction model & 74.098 & 2 & 37.049 & 6.644 & 0.005 \\
Pre-test & 72.898 & 1 & 72.898 & & \\
Group & 5.31 & 1 & 5.31 & 13.072 & 0.001 \\
Error & 150.569 & 27 & 5.577 & & \\
Total & 13038 & 30 & & 0.952 & 0.338 \\
\hline
\end{tabular}

Table 3. Results of the covariance analysis test to examine the effect of two educational methods on basketball dribbling skill

\begin{tabular}{llllll}
\hline Source & SS & df & MS & F & Sig. \\
\hline Correction model & 72.919 & 2 & 36.459 & 53.198 & 0.001 \\
Pre-test & 72.872 & 1 & 72.872 & 106.329 & 0.001 \\
Group & 0.16 & 1 & 0.16 & 0.632 & 0.234 \\
Error & 18.504 & 27 & 0.685 & & \\
Total & 3789.944 & 30 & & & \\
\hline
\end{tabular}

Table 4. Results of the covariance analysis test to investigate the effect of two educational methods on basketball shot skill

\begin{tabular}{llllll}
\hline Source & SS & df & MS & F & Sig. \\
\hline Correction model & 473.538 & 2 & 236.769 & 7.748 & 0.002 \\
Pre-test & 126.738 & 1 & 126.738 & 4.147 & 0.001 \\
Group & 432.208 & 1 & 432.208 & 14.143 & 0.001 \\
Error & 825.129 & 27 & 30.56 & & \\
Total & 32662 & 30 & & & \\
\hline
\end{tabular}


technique, while the traditional training group did not make any progress. These results are consistent with the findings of Nathan and Haynes [20] and inconsistent with the findings of French et al. [19]. A possible reason for these conflicting results may be the type of selected game.

In the French and colleagues' research, the impact of tactic-based methods and the technique for learning was done in badminton play [19]. The badminton game in Bunker and Thorup division [5] is part of the net/wall game, but basketball in the game is part of an attack game, like hockey in Nathan and Haynes [20]. The PP group performed better than the traditional group in post-test of shots and pass technique. These results are inconsistent with more research findings $[4,10,12,27]$. These results are consistent with the content of the tactical group training program in this study. The overall content of the tactics-based training program included warming up, skill drilling, and game play that after the ninth session changed to moderated games. Skill drilling is the most part of the training sessions, especially at the initial sessions, is given in static and unrestricted conditions. It seems that these conditions are very much in line with the requirements for executing skill tests such as basketball performance one. It seems that the skill drilling and practical skill at the end of each session has also been effective in the tactical progress of this group. But why the groups did not make a significant difference in terms of shooting test is interesting.

Participants at all stages of the warm-up phase and the interruptions between the games repeatedly practiced shooting technique and they constantly practiced it. Possibly, the frequent repetition of this technique by the participants may have made the groups differently meaningless. A review of the trend shows that both educational approaches have contributed to improved basketball performance.

As Thomas and Thomas stated, selection of the appropriate response or making appropriate choices in sport does not necessarily correlate with successful performance [28]. In accordance with the PP model, participants first develop game understanding and decision making, and later develop technical skills. There was no progress in dribbling performance; the relatively weak decision making by the PP group in the post-test was influenced by the weakness of the group in performing of the skill. Since technical weaknesses may limit the choices that are made in the situations of the game. In different situations, the target selection may be done correctly, but the allocation of the appropriate parameters to the chosen motor program is a basic problem for beginners [29], or, as Turner et al. assumes, the two processes, "What to do" and "How" is likely to be specific to complex motor skills such as playing [12]. The relatively long protocol of this study may have been able to improve the weak section of "How", in post-test of PP group. However, in the field of pass technique performance and enhancement program of it after ninth session in PP group, is likely to improve the performance of the group. Perhaps this has led to a significant improvement in the PP group from pre-test to post-test.

Another important aspect of improving the performance of the pass in the PP group is the use of the question and answer training principle. As Pra'xedes et al. concluded, the use of the principle of questionand-answer questions in the field of moderated games makes decision-making progress [23]. Raab and Johnson emphasized that "the question" is a tool that can succeed in very complex situations because it leads the athlete's attention to important aspects of the game and receives positive results [30]. Similar results have been reported in other studies comparing technique-based and gamebased groups $[8,10,11]$. But others have reported PP's superiority in performing skill [4, 13, 14, 23].

Despite the superiority of PP group in pass and shot performance tests, these groups did not have a particular advantage in terms of skill performance in dynamic game play than the traditional group. Miller [15] concluded in a review article that there is a relationship between the duration of the game-based intervention and communication skill performance. In the present study, the groups trained for 18 sessions and each session for one hour. This volume of interventions seems to have been effective and promotes groups from pre-test to posttest. In the analysis and comparison of group changes in terms of shot and pass, the results showed that PP practice protocols have promoted performance.

\section{Conclusion}

The results reported in other similar studies [4, $8,9,16]$. Contrary to skill performance, the support performance in game-based models can be developed faster. According to Miller's conclusion, support in the game can also be developed in tactics based on short term training (8 sessions) [15]. In the game-based protocol in this study, 4 sessions (sessions 6, 7, 8, and 9) were devoted to the development of tactical awareness. The significant difference between the game-based group and the traditional one is in line with Miller's deduction in the mid-test [15]. It seems that the exercises of these groups, based on moderated questions and games, have led to the development of cognitive and decision-making skills. This development in decision making is so deep that even in relatively more complex situations of basketball full games, game-based groups can take advantage of it. Considering the process of questioning in the form of moderated games as an effective and influential factor in decision-making, the PP team benefited from this process during the training program. But in connection with the traditional group, even the inclusion of training programs did not lead to the development of dribbling and pass technique during the game. However, the use of moderated games and the use of tactical strategies after the ninth session for the traditional group may not be fully appreciated by McMurray's proposal technique [22] Technique-to recognition. As Pra'xedes et al. suggested [23], it seems the use of the question in the form of moderated games seems to be the most prominent feature of the PP model, which can, in particular, enhance decision 
making in the game. It is suggested that a similar study would be conducted over a long period of time with more sample sizes in different age domains. It is also suggested that the impact of these models should be considered on psychological outcomes such as the motivation of continuing training, enjoyment of practice among youth.

\section{References}

1. Butler JI, Griffin LL. More Teaching Games for Understanding: Moving Globally. Human Kinetics; 2010.

2. Williams AM, Hodges NJ. Practice, instruction and skill acquisition: Challenging tradition. Journal of Sports Sciences. 2005; 6: 637-50.

3. Hastie P. Applied benefits of the sport education model. Journal of Physical Education, Recreation, and Dance. 1998; 69(4): 24-26.

4. Pritchard T, Hawkins A, Wiegand R, Metzler J N. Effects of Two Instructional Approaches on Skill Development, Knowledge, and Game Performance. Measurement in Physical Education and Exercise Science. 2008; 12: 219236.

5. Bunker D, Thorpe R. A model for the teaching of games in secondary schools. Bulletin of PhysicalEducation, 1982; 18(1): 5-8.

6. Rink JE, French KE, Tjeerdsma B L. Foundations for the learning and instruction of sport and games. Journal of Teaching in Physical Education, 1996; 15: 399-417.

7. Holt J. Ward P., Wallhead T. The Transfer of Learning from Play Practices to Game Play in Young Adult Soccer Players. Physical Education and Sport Pedagogy, 2006; 11 (2): 101118.

8. Gray S, Sproule J. Developing pupils' performance in team invasion games. Physical Education and Sport Pedagogy, 2011; 16: 15-32.

9. Gray S, Sproule J, Morgan K. Teaching Team Invasion Games and Motivational Climate. European Physical Education Review, 2009; 15 (1): 65-89.

10.Tallir I, Lenoir M, Valcke M, Musch E. Do alternative instructional approaches result in different game performance learning outcomes? Authentic assessment in varying game conditions. International Journal of Sport Psychology, 2007;38(3):263-282.

11. Chatzopoulos D, Drakou A, Kotzamanidou M, Tsorbatzoudis H. Girls' soccer performance and motivation: Games vs technique approach. Perceptual and Motor Skills, 2006; 103 (2): 463-470.

12.Turner A, Martinek T. An investigation into teaching games for understanding: Effects on skill, knowledge and game play. Research Quarterly for Exercise and Sport, 1999; 70 (3): 286-296.

13.Mesquita I, Farias C, Hastie P. The Impact of a Hybrid Sport Education-Invasion Games Competence Model Soccer Unit on Students' Decision Making, Skill Execution and Overall Game Performance. European Physical Education Review, 2012; 18 (2): 205-219.

14.Zeatou A, Vernadakis N, Derri V, Bebetsos E, Filipou F. The effect of game for understanding on backhand tennis skill learning and self-efficacy improvement in elementary students. Procedia-Social and Behavioral Sciences. 2014; 152:765-771.

15.Miller A. Games Centered Approaches in Teaching Children \& Youth: Systematic Review of Associated Student Outcomes. Journal of Teaching in Physical Education, 2015; 34: $36-58$

16.Harvey S, Cushion C, Wegis H, Massa-Gonzalez A. Teaching

\section{Acknowledgments}

I would like to express my deepest appreciation to all those who provided me the possibility to complete this research.

\section{Conflict of interests}

The authors state that there is no conflict of interest.
Games for Understanding in American High-School Soccer: A Quantitative Data Analysis Using the Game Performance Assessment Instrument. Physical Education and Sport Pedagogy. 2010; 15 (1): 29-54.

17.Lee M A-h, Ward P. Generalization of Tactics in Tag Rugby from Practice to Games in Middle School Physical Education. Physical Education and Sport Pedagogy. 2009; 14 (2): 189-207.

18.McMorris T. Teaching games for understanding: its contribution to the knowledge of skill acquisition from a motor learning perspective, European Journal of Physical Education. 1998; 3: 65-74

19.French K, Werner P, Hussey K, Taylor K, Jones J. The effects of a 6-week unit of tactical, skill, or combined tactical and skill instruction on badminton performance of ninth-grade students. Journal of Teaching in Physical Education, 1996; 15(4): 439-463.

20.Nathan S, Haynes J. A move to an innovative games teaching model: Style E Tactical [SET). Asia-Pacific Journal of Health, Sport \&. Physics Education, 2013; 4(3): 287-302.

21.Harvey S, Jarrett K. A review of the game-centred approaches to teaching and coaching literature since 2006. Physical Education and Sport Pedagogy, 2013; 30: 1-23.

22.McMurray B, Horst JS, Toscano JC, Samuelson LK. Integrating connectionist learning and dynamical systems processing: Case studies in speech and lexical development. In: Spencer JP, Thomas MSC, McClelland JL, editors. Toward a new grand theory of development: Connectionism and dynamic systems theory reconsidered. New York: Oxford University Press; 2009. pp. 218-252.

23.Pra'xedes A, Moreno A, Sevil J, Garci'a-Gonza'lez L, Villar D F. A Preliminary Study of the Effects of a Comprehensive Teaching Program, Based on Questioning, to Improve Tactical Actions in Young Footballers. Perceptual and Motor Skills, 2016; 122(3): 742-756.

24.Holt N L, Strean W B, Bengoechea E G. Expanding the teaching games for understanding model: New avenues for future research and practice. Journal of Teaching in Physical Education, 2002; 21(2): 162-176.

25.Hadavi F, Farahani A, Eezadi A. Measurement, Deliberation and Evaluation in Physical Education. Tehran: Hatmi; 2013.

26. Oslin J, Mitchell S, Griffin L. The Game Performance Assessment Instrument [GPAI): Development and Preliminary Validation. Journal of Teaching in Physical Education, 1998; 17: 231-243.

27.Alison S, Thorpe R. Comparison of the effectiveness of two approaches to teaching games within PE. A skills approach verses a games for understanding approach. British Journal of Physical Education, 1997; 28(3): 9-13.

28.Thomas K, Thomas JR. Developing expertise in sport: The relation of knowledge and performance. International journal of sport psychology, 1994; 25: 295-312.

29.McPherson S L. The development of sport expertise: Mapping the tactical domain. Quest. 1994; 46: 223- 240.

30.Raab M, Johnson M. Implicit learning as a means to intuitive decision making in sports. In H. Plessner, T. Betsch, \& C. Betsch (Eds.), Intuition in judgment and decision making, London, England: Routledge; 2007. P. 119-134. 
Information about the authors:

Ghanati P. (Corresponding Author); MA student of Motor Behavior; http://orcid.org/0000-0001-8468-2915; Parinazghanati90@gmail. com; Department of Physical Education and Sport Sciences, Urmia University; West Azerbaijan Province, 24، Urmia Lake Bridge, Iran.

MohammadZadeh H.; Prof.; http://orcid.org/0000-0002-9345-2158; ha.moha64@gmail.com; Department of Physical Education and Motor Behavior, Urmia University; West Azerbaijan Province, 24، Urmia Lake Bridge, Iran.

Cite this article as: Ghanati P, MohammadZadeh H. Comparison of the effect of game based on educational method and traditional approach on the performance of selected basketball skills. Physical education of students, 2018;22(4):175-181. doi:10.15561/20755279.2018.0402

The electronic version of this article is the complete one and can be found online at: http://www.sportedu.org.ua/index.php/PES/issue/archive

This is an Open Access article distributed under the terms of the Creative Commons Attribution License, which permits unrestricted use, distribution, and reproduction in any medium, provided the original work is properly cited (http://creativecommons.org/licenses/by/4.0/deed.en).

Received: 11.06 .2018

Accepted: 09.07.2018; Published: 23.08.2018 